\title{
The First Case Report of Robot-Assisted Fluoroscopy-Guided Renal Access During Endoscopic Combined Intrarenal Surgery
}

\author{
Kazumi Taguchi, MD, PhD, Shuzo Hamamoto, MD, PhD, Kengo Kawase, MD, \\ Tatsuya Hattori, MD, Tomoki Okada, MD, Ryosuke Chaya, MD, Takashi Nagai, MD, \\ Taiki Kato, MD, PhD, Atsushi Okada, MD, PhD, and Takahiro Yasui, MD, PhD
}

\begin{abstract}
Background: During percutaneous nephrolithotomy (PCNL) and endoscopic combined intrarenal surgery (ECIRS), obtaining renal access is the most critical step to achieving effective treatment without major intraoperative complications. Among a variety of methods attempted to improve the access, robot-assisted fluoroscopy-guided (RAFG) renal access has been introduced to mitigate technical human errors and overcome challenging learning curves. In this study, we present our first experience with an automated needle targeting with an X-ray (ANT-X) device for minimally invasive (mini-) ECIRS.

Case Presentation: A 75-year-old healthy woman with a $6.0 \mathrm{~cm}^{3}$ left kidney stone was referred to our hospital for surgical treatment. The patient underwent mini-ECIRS utilizing RAFG renal access without complication, and the stone was completely removed. The ureteral stent and transurethral catheter were removed on postoperative day 2 , and the patient was discharged on postoperative day 3 . There were no residual fragments detected by CT as of 3 months after the surgery.

Conclusion: To our knowledge, this is the first report of the effective use of RAFG mini-ECIRS for a kidney stone. The overall outcome was positive, indicating the feasibility of ANT-X use for PCNL and ECIRS.

Keywords: percutaneous nephrolithotomy, endoscopic combined intrarenal surgery, miniendoscopic intrarenal surgery, robot-assisted fluoroscopy-guided, artificial intelligence

\section{Introduction}

$\mathbf{P}$ ROPER RENAL ACCESS during percutaneous nephrolithotomy (PCNL) is key to effective treatment by facilitating stone fragmentation and reducing intraoperative complications. A variety of modalities have been applied to gain optimal renal access, such as minimally invasive endoscopic tools, ultrasound guidance, real-time ultrasonography, and ureteroscopic assistance. ${ }^{1}$

However, there remain concerns regarding the technical difficulty of this step, in particular for those who are not well trained or experienced, as it requires considerable practice. Even during an endoscopic combined intrarenal surgery (ECIRS), in which ureteroscope use assists percutaneous access as well as stone fragmentation, observation of a renal collecting system filled by a stone is not always easy. To overcome these learning curve issues, we have utilized an automated needle targeting with X-ray (ANT-X) system (NDR Medical Technology, Singapore) to provide optimal and safe renal access. ${ }^{2}$

The Johns Hopkins URobotics laboratory first developed robot-assisted renal puncture in 2002. The renal puncture process was automated by integrating it with the fluoroscopic imaging using a remote controller. They further upgraded the system by mounting it on a CT scanner for better quality of ideal puncture calculated by scanned images. In addition, they invented Mrbot, a robot system integrated with magnetic resonance imaging for transperitoneal prostate biopsy, approved by the Food and Drug Administration. ${ }^{3}$

More recently, there has also been a surge of artificial intelligence (AI) applications for the medical field, and among these recent AI and robotic advancements, ANT-X has been introduced as a robot-assisted device for PCNL. It includes a robotic metal arm to fix and move the angle of the percutaneous needle as well as AI software that analyzes an optimal puncture angle from captured fluoroscopic images. Both benchtop and animal studies have confirmed the feasibility and comparability between ANT-X and conventional fluoroscopy/ ultrasound guidance for percutaneous renal access.
\end{abstract}

Department of Nephro-urology, Nagoya City University Graduate School of Medical Sciences, Nagoya, Japan. 
In this study, we present our first experience with effectively removing a left pelvic stone in a minimally invasive (mini-) ECIRS case by robot-assisted fluoroscopy-guided (RAFG) renal access using ANT-X. The outcome of this case was acceptable with a short surgical and fluoroscopic duration and without any major complications.

\section{Case Presentation}

\section{Clinical history and diagnosis}

A 75-year-old woman was referred to our hospital for surgical treatment of a $6.0 \mathrm{~cm}^{3}$ left kidney stone that was detected on routine examination. She was taking medication for hypertension and had a medical history of blood transfusion-related hepatitis; otherwise, she was physically and mentally healthy without any symptoms, including fever, pain, or gross hematuria. Preoperative urinary analysis showed slight pyuria, but the urinary culture was negative. Laboratory testing confirmed that her white blood cell count was $7500 / \mu \mathrm{L}$, serum C-reactive protein level was $0.15 \mathrm{mg} / \mathrm{dL}$, creatine level was $0.67 \mathrm{mg} / \mathrm{dL}$, calcium level was $9.5 \mathrm{mg} / \mathrm{dL}$, phosphate level was $3.2 \mathrm{mg} / \mathrm{dL}$, and intact-parathyroid hormone level was $27 \mathrm{pg} / \mathrm{mL}$. Her liver enzyme level was slightly above normal at $50 \mathrm{U} / \mathrm{L}$ and $59 \mathrm{U} / \mathrm{L}$ of aspartate aminotransferase and alanine aminotransferase, respectively.

Kidney-ureter-bladder radiograph and multislice CT revealed a left ureteropelvic junction stone (size $21 \times 20 \times 14 \mathrm{~mm}^{3}$; CT value $1288 \mathrm{HU}$ ) with a lower posterior caliceal fragment (size $6 \times 5 \times 4 \mathrm{~mm}^{3}$; CT value $1095 \mathrm{HU}$ ). We further performed a three-dimensional $\mathrm{CT}$ of her kidney to evaluate the detailed anatomy of the stone and renal collecting system (Fig. 1).

\section{Surgical technique}

After discussion of treatment options including retrograde intrarenal surgery, mini-PCNL, and mini-ECIRS between the patients and surgery team, she preferred mini-ECIRS because of a potential higher stone-free rate and shorter duration of ureteral stent placement than the others. After obtaining written consent for the surgery itself and for the patient's participation in the clinical research (No. 2019A002) regarding RAFG renal access, we performed mini-ECIRS with the patient in the split-leg prone position under general anesthesia.

Based on the preoperative CT images, upper pole puncture was planned for this case. The puncture point was marked on the skin between the 11th and 12th ribs; thereafter, the ANT-X device was set up over the patient (Fig. 2). We simultaneously inserted a flexible cystoscope $\left(\right.$ CYF-VHA ${ }^{\circledR}$, Olympus, Tokyo, Japan) and placed a 10/12F ureteral access sheath (Bi-Flex ${ }^{\circledR}$, ROCAMED, Monaco) up to the ureteropelvic junction through a guidewire (ENDO ACCESS ${ }^{\circledR}$, BD, Franklin Lakes, NJ). Then, a flexible ureteroscope (URF-V2 ${ }^{\circledR}$, Olympus) was inserted through the access sheath and passed beside the stone to the upper calix to establish artificial hydronephrosis and observe the puncture site on the papilla. After injecting contrast medium from the ureteroscope, the ANT-X software captured the fluoroscopic image of the renal collecting system and set an ideal alignment for the puncture. Under the navigation of the ANT-X software, the surgeon inserted an 18-gauge needle into the upper calix and placed the guidewire after confirming the needle tip on the papilla under ureteroscopic direct vision as described by Khan et al. ${ }^{4}$ Under endoscopic guidance, the tract was dilated with the inner set of the ureteral access sheath; then a 16.5/17.5F metal sheath was placed as the tract (Fig. 3).

The stones were fragmented by both a pneumatic lithotripter (LithoClast $^{\circledR}$, Boston Scientific, Marlborough, MA) with a 12F nephroscope (Karl Storz, Tuttlingen, Germany) and a Ho:YAG laser (Cyber Ho ${ }^{\circledR}$, Quanta system, Milan, Italy) with a ureteroscope. We completely extracted the fragments from the minipercutaneous tract with the vacuum effect, assisted by irrigation from the ureteroscope. At the end of the procedure,
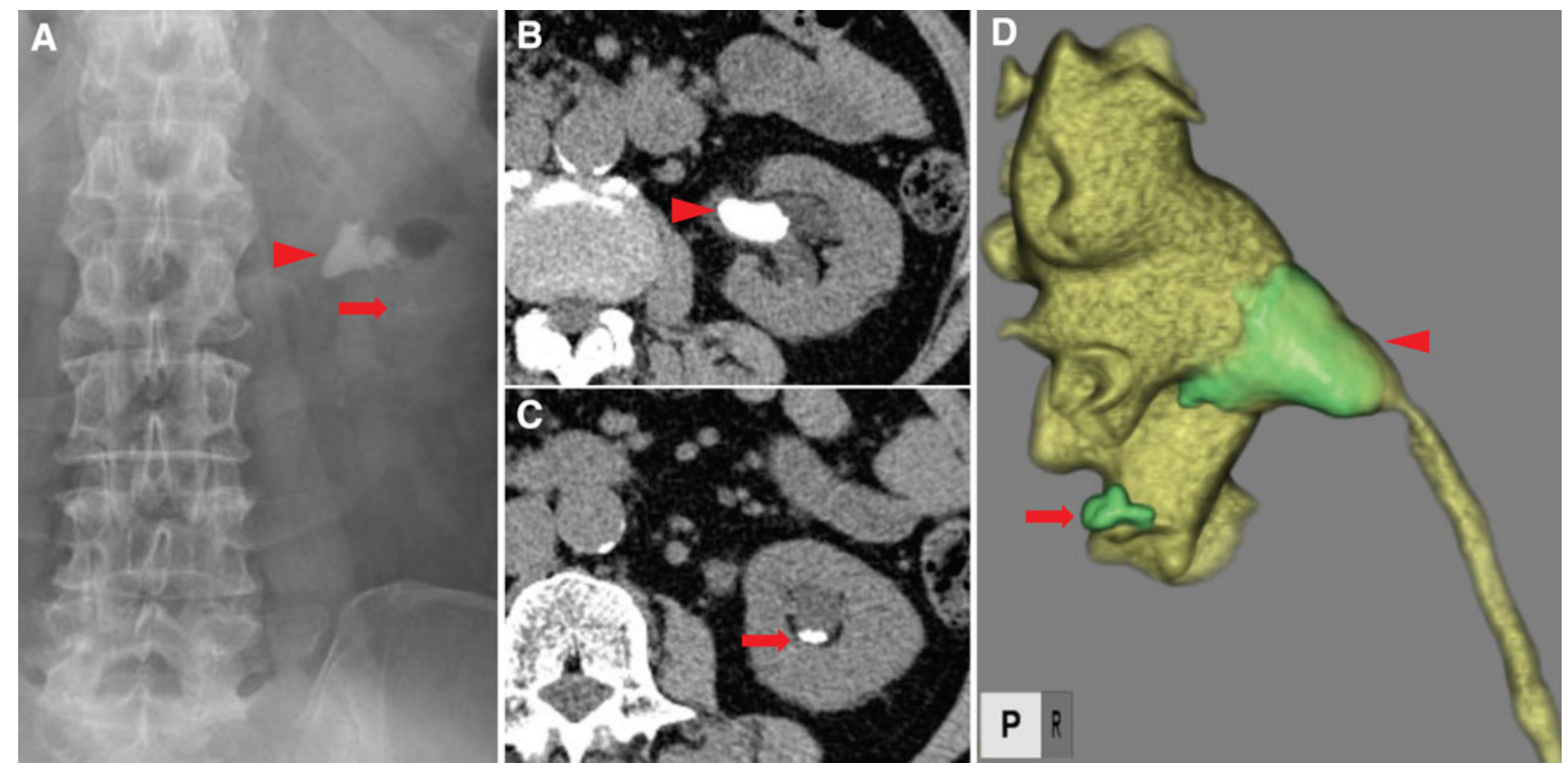

FIG. 1. Preoperative kidney-ureter-bladder radiograph (A) and CT (B-D) demonstrate the presence of large left pelvic (arrowheads) and small lower calix (arrows) stones. 
FIG. 2. Intraoperative images of the ANT-X device over the patient (A) and a fluoroscopic view of the renal collecting system (B). Arrows $=$ flexible ureteroscope, arrowheads $=$ ANT-X device. ANT-X, automated needle targeting with an X-ray.
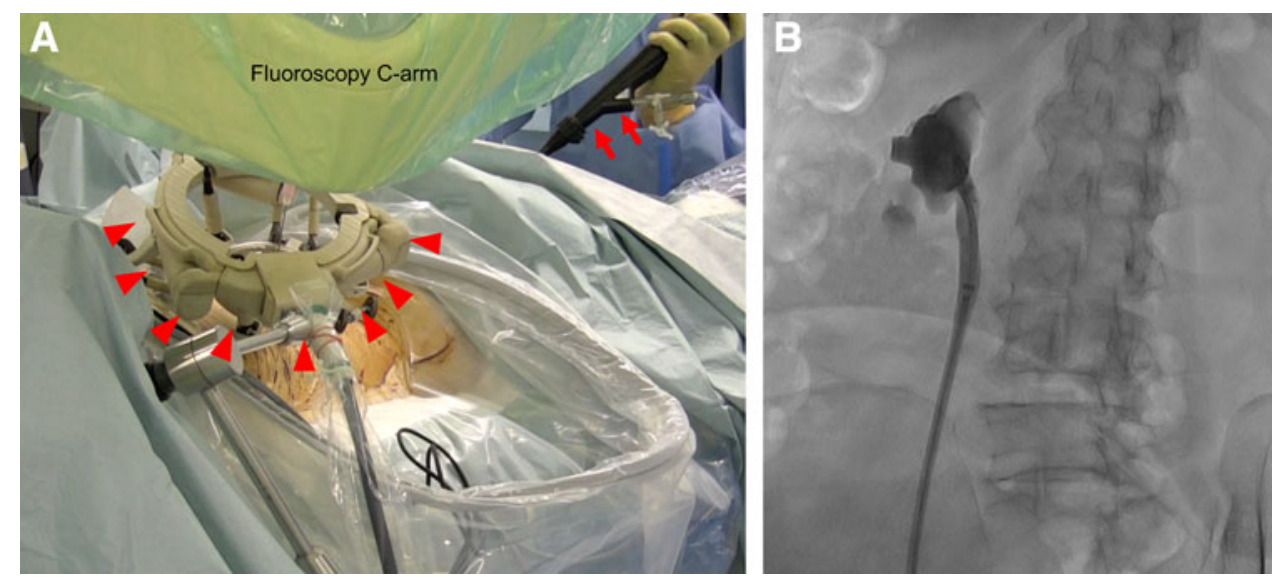

a 4.8F ureteral stent $\left(\right.$ Tria $^{\circledR}$, Boston Scientific) was placed and connected with a $14 \mathrm{~F}$ transurethral catheter.

\section{Operative results}

The total procedure time was 54 minutes, including 5 minutes for device setup and 3 minutes for puncturing time. Fluoroscopy duration was 6 minutes and 4 seconds. The ureteral stent and transurethral catheter were both removed on postoperative day 2 , and the patient was discharged from the hospital on postoperative day 3. Her serum levels of hemoglobin decreased from 13.8 to $12.8 \mathrm{~g} / \mathrm{dL}$, and her creatinine level remained unchanged on postoperative day 1 . There were no major perioperative complications. The stone composition was $78 \%$ calcium oxalate $(90 \%$ and $10 \%$ of calcium oxalate monohydrate and dihydrate, respectively) and $22 \%$ calcium phosphate. The CT image 3 months after surgery showed no residual fragment or hydronephrosis in the kidney (Fig. 4).
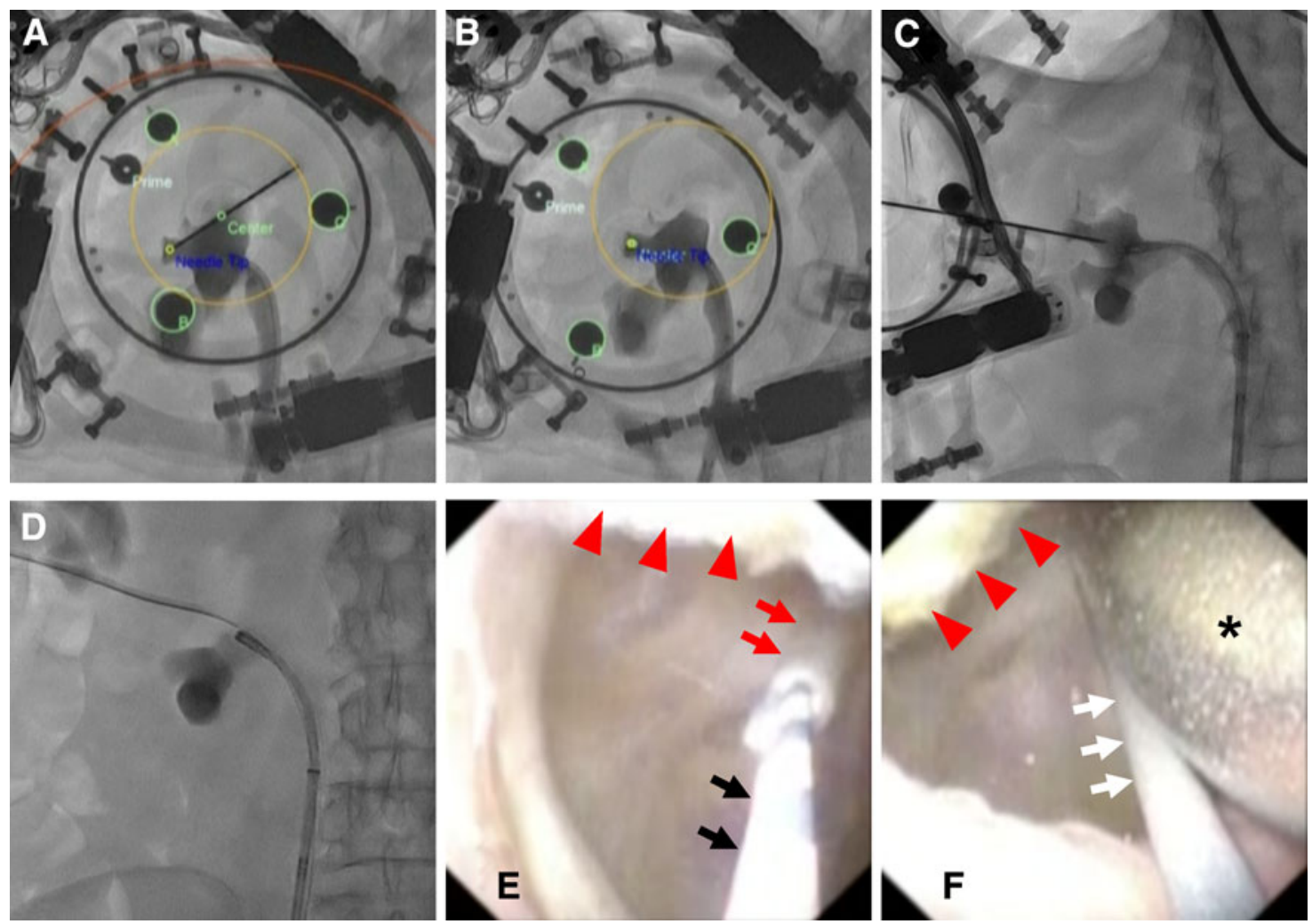

FIG. 3. Fluoroscopy-integrated views of the device software (A, B), fluoroscopic images (C, D), and ureteroscopic images (E, F) during renal access. (A) After adjusting the device location by confirming the marker (three green circles) positions, the needle tip selection was completed. (B) The overlap of the needle tip and shaft as a bull's eye indicated that the calculation of the puncture alignment was finished. (C) With the C-arm angle tilted, the needle was inserted into the left upper calix. (D, E) The guidewire was passed through the inner set of the ureteral access sheath with direct vision from the flexible ureteroscope. (F) The metal sheath was inserted as the renal access tract. Red arrowheads=pelvic stone, red arrows $=$ inner set of ureteral access sheath, black arrows $=$ guidewire for renal access, white arrows $=$ safety guidewire, asterisk $=$ metal sheath. 

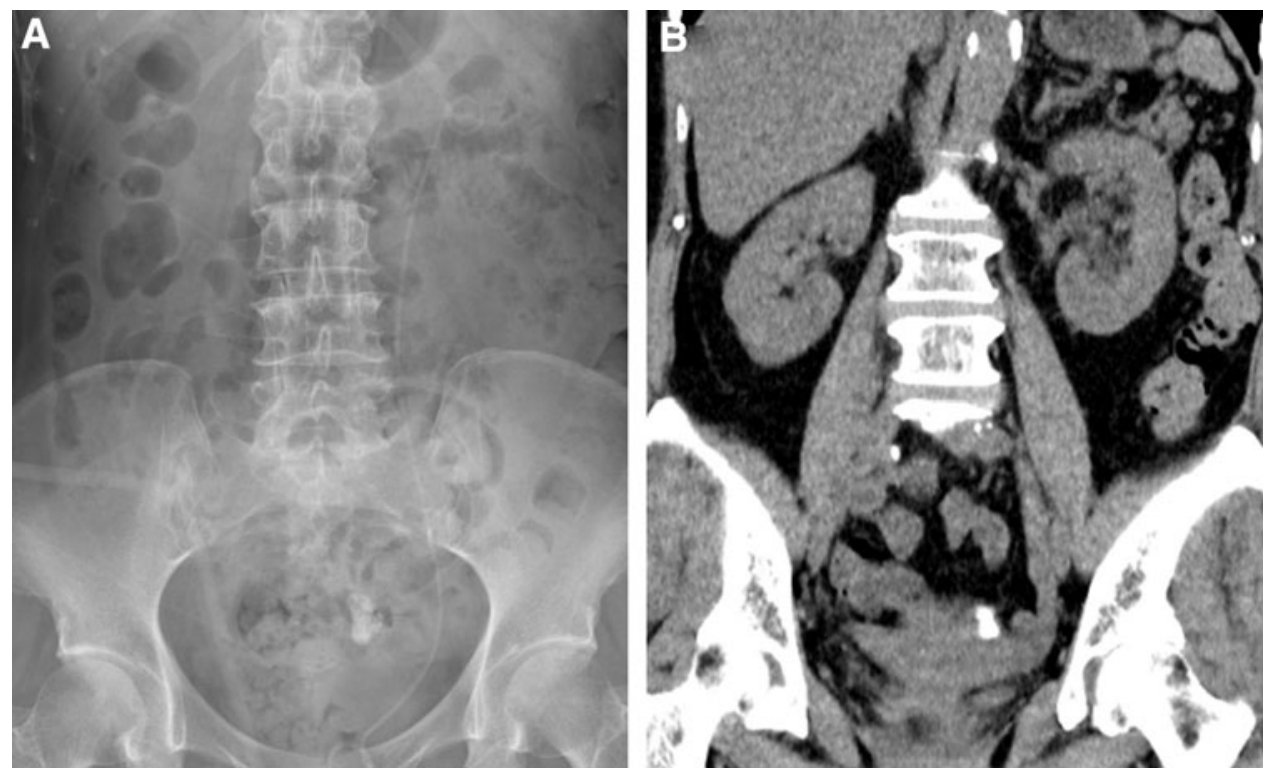

FIG. 4. Postoperative kidney-ureter-bladder radiograph on the day after surgery (A) and CT 3 months after surgery (B) demonstrate no residual fragment in the left kidney.

\section{Discussion}

ECIRS has become one of the standard procedures for the treatment of large kidney stones. It utilizes a simultaneous approach by a nephroscope and a flexible ureteroscope. ${ }^{5}$ There are some merits of double scopes, such as ureteroscopeassisted renal access and facilitation of fragmentation for different calices. Despite these advantages, coordination with two surgeons (PCNL and ureteroscopy side) is sometimes challenging, and puncture of the targeted renal calix is still the most important process that substantially influences surgical outcomes. Like other urologists, we have tried to develop reliable renal punctures by utilizing miniaturized sheaths and real-time virtual sonography. However, we still have had difficulty improving our skills and, unfortunately, have experienced serious complications such as renal injury and severe bleeding.

In this study, we present our first experience with effectively removing a $6.0 \mathrm{~cm}^{3}$ pelvic stone during a minimally invasive (mini-) ECIRS case by RAFG renal access using ANT-X. Although the outcome of the case was positive, the key question is how feasible these technologies will be and how soon they will be available for clinical use.

Surprisingly, some aspects of RAFG puncture have been positive, even for those who recently began using fluoroscopic guidance, like us. Although we had little experience performing fluoroscopy-guided PCNL, we could safely complete our first trial of RAFG renal access. Based on our earlier experiences, we were comfortable performing miniECIRS because of its safety and effectiveness for treating large kidney stones. Visual and irrigation support from a ureteroscope also facilitated wire insertion and dilatation of the tract after the papilla puncture. Therefore, we first tried to assess the feasibility of RAFG renal access during ECIRS rather than PCNL. The outcome of this case was acceptable with short surgical and fluoroscopic duration without any major complications. Although this trial was performed on the relatively small-sized stone with uncomplicated renal collecting system, these findings could encourage us to apply this new modality for PCNL toward versatile stones, including staghorn stones.
However, some issues need to be discussed for the wide use of RAFG renal access. For example, the cost of installing ANT$\mathrm{X}$ device and cooperation can weigh an additional burden on hospitals and operating room staff. In addition, the function of this device is for navigation of the ideal angle to puncture but not for the automation of puncture itself. Therefore, the manual needle insertion needs to be performed by urologists.

\section{Conclusion}

We have utilized the RAFG mini-ECIRS for the removal of a $6.0 \mathrm{~cm}^{3}$ left kidney stone. To our knowledge, this is the first report of the application of ANT-X for renal puncture during ECIRS. The overall outcome was safe and effective, indicating the feasibility of RAFG ECIRS. We believe this procedure could become a feasible option for both PCNL and ECIRS.

\section{Acknowledgments}

We thank NDR Medical Technology for providing the ANT-X device and helping us to install the equipment in our hospital as well as radiology staff and medical engineers to coordinate and apply the RAFG technique to our operation room.

\section{Disclosure Statement}

No competing financial interests exist.

\section{Funding Information}

No funding was received for this article.

\section{References}

1. Tailly $\mathrm{T}$, Denstedt J. Innovations in percutaneous nephrolithotomy. Int J Surg 2016;36:665-672.

2. Taguchi K, Hamamoto S, Okada A, et al. Robot-assisted fluoroscopy versus ultrasound-guided renal access for nephrolithotomy: A Phantom Model Benchtop Study. J Endourol 2019;33:987-994. 
3. Stoianovici D, Kim C, Petrisor D, et al. MR safe robot, FDA clearance, safety and feasibility prostate biopsy clinical trial. IEEE ASME Trans Mechatron 2017;22:115-126.

4. Khan F, Borin JF, Pearle MS, et al. Endoscopically guided percutaneous renal access: "Seeing is believing." J Endourol 2006;20:451-455.

5. Taguchi K, Cho SY, Ng AC, et al. The Urological Association of Asia clinical guideline for urinary stone disease. Int J Urol 2019;26:688-709.

Address correspondence to: Shuzo Hamamoto, MD, PhD Department of Nephro-urology Nagoya City University Graduate School of Medical Sciences 1 Kawasumi, Mizuho-cho, Mizuho-ku Nagoya 467-8601 Japan

E-mail: hamamo10@med.nagoya-cu.ac.jp

$\begin{aligned} & \text { Abbreviations Used } \\ & \mathrm{AI}=\text { artificial intelligence } \\ & \mathrm{ANT}-\mathrm{X}=\text { automated needle targeting with an X-ray } \\ & \mathrm{CT}=\text { computed tomography } \\ & \mathrm{ECIRS}=\text { endoscopic combined intrarenal surgery } \\ & \mathrm{HU}=\text { Hounsfield unit } \\ & \mathrm{PCNL}=\text { percutaneous nephrolithotomy } \\ & \mathrm{RAFG}=\text { robot-assisted fluoroscopy-guided }\end{aligned}$

Cite this article as: Taguchi $\mathrm{K}$, Hamamoto S, Kawase K, Hattori T, Okada T, Chaya R, Nagai T, Kato T, Okada A, Yasui $T$ (2020) The first case report of robot-assisted fluoroscopy-guided renal access during endoscopic combined intrarenal surgery, Journal of Endourology Case Reports 6:4, 310-314, DOI: 10.1089/ cren.2020.0125. 